\title{
Swertiamarin suppresses proliferation, migration, and invasion of hepatocellular carcinoma cells via negative regulation of FRAT1
}

\author{
Shufeng Xiao, ${ }^{1,2}$ Haoran Tang, ${ }^{3}$ Yao Bai, ${ }^{4}$ Renchao Zou, ${ }^{1}$ Zongfang Ren, ${ }^{5}$ Xuesong Wu, ${ }^{3}$ Zhitian Shi, ${ }^{1}$ Song Lan, \\ Wei Liu, ${ }^{7}$ Tiangen Wu, ${ }^{1}$ Cheng Zhang, $^{1}$ Lin Wang ${ }^{1}$ \\ ${ }^{1}$ Department of Hepatobiliary Surgery, the Second Affiliated Hospital of Kunming Medical University, Kunming \\ ${ }^{2}$ Department of General Surgery, Puer People's Hospital, Puer \\ ${ }^{3}$ Department of Gastroenterological Surgery, the Second Affiliated Hospital of Kunming Medical University, Kunming \\ ${ }^{4}$ School of Medicine, Yunnan University, Kunming \\ ${ }^{5}$ Department of Critical Care Medicine, the Second Affiliated Hospital of Kunming Medical University, Kunming \\ ${ }^{6}$ Department of Pathology, Puer People's Hospital, Puer \\ ${ }^{7}$ Department of Hepatobiliary Surgery, The People's Hospital of Chuxiong Yi Autonomous Prefecture, the Fourth \\ Affiliated Hospital of Dali University, Chuxiong, China
}

\begin{abstract}
Studies have shown that swertiamarin (STM) has multiple biological activities, but its antitumour effects and molecular mechanisms are still unclear. The present research aimed to validate the STM's impacts on the proliferation, migration, and invasion of hepatocellular carcinoma (HCC) cells, and to study its potential mechanism. Two HCC cell lines were treated with STM. Tumour growth was observed by the mouse tumour xenografts model. HCC cell lines stably expressing frequently rearranged in advanced T-cell lymphomas 1 (FRAT1) were generated by lentivirus-mediated overexpression. Cell viability, proliferation, migration, and invasion were observed using Cell Counting Kit-8 (CCK8), the xCELLigence Real-Time Cell Analyzer system (RTCA), and transwell analysis, respectively. Quantitative real-time polymerase chain reaction (qRT-PCR) and Western blotting were used to observe the expression of FRAT1 and proteins related to the Wnt// -catenin signalling pathway. Tumour growth was inhibited by STM in vivo. STM suppressed the proliferation, migration, and invasion of HCC cells. STM negatively regulated FRAT1 expression, whereas overexpressed FRAT1 blocked the antitumour function of STM. The results revealed that STM suppressed the FRAT1/Wnt/ $\beta$-catenin signalling pathway. The findings of this study provide new insights into investigation of therapeutic strategies against HCC.
\end{abstract}

Key words: Swertiamarin; FRAT1; HCC; proliferation; migration; invasion.

Correspondence: Dr. Lin Wang, Department of Hepatobiliary Surgery, the Second Affiliated Hospital of Kunming Medical University, 374 Yunnan-Burma Avenue, Kunming 650101, China. Tel. +86.0871.65352087. E-mail: linwang705@gmail.com

Contributions: SX and HT contributed equally to this study; LW and SX designed the experiments; HT, YB and RZ completed the experiments; ZR, XW, TW and CZ shared equipment and reagents; ZS, SL and WL analyzed the data; SX and HT wrote the manuscript.

Conflict of interest: The authors declare that they have no competing interests, and all authors confirm accuracy.

Funding: This research was funded by the Yunnan Hepatobiliary and Pancreatic Disease Clinical Center Fund and the National Natural Science Foundation of China (No. 81660399, No. 81860423).

Availability of data and materials: The data used to support the findings of this study are available from the corresponding author upon request.

Ethical Approval: All procedures involving animals were carried out in accordance with the Policy on the Care and Use of Animals by the Ethical Committee and this study was approved by the Administration Committee of Experiment Animals of The Second Affiliated Hospital of Kunming Medical University, 374 Yunnan-Burma Avenue, Kunming Yunnan 650101, China (LLSC-2020052901). 


\section{Introduction}

Liver cancer is the sixth most frequent tumor type worldwide and the fourth most frequent type of cancer-related decease. ${ }^{1}$ Conventional treatments like surgical resection, local resection, liver transplantation, chemotherapy, and radiation therapy are usually ineffective because most patients are diagnosed with advanced disease, after the optimal surgical window or have a poor prognosis. $^{2,3}$ Therefore, pharmaceutical drugs and treatment options are indispensable for hepatocellular carcinoma (HCC) therapy, especially for advanced-stage patients. Natural products and their derivatives are important sources of new drugs, and candidate cancer treatment methods, such as anticancer agents, are receiving increased attention. ${ }^{4}$

Swertiamarin (STM), $\mathrm{C}_{16} \mathrm{H}_{22} \mathrm{O}_{10}$, is a bitter secoiridoid glycoside and is the main biologically active component of Gentianaceae plants. STM has various kinds of pharmacological actions, including antimicrobial, ${ }^{5}$ antifungal, ${ }^{6}$ anti-inflammatory, ${ }^{7}$ antidiabetes, ${ }^{8}$ hepatoprotective, ${ }^{9}$ and anti-HBV (hepatitis B virus) ${ }^{10}$ activities. Although some studies were conducted on the anticancer effects of total glycosylates containing STM extracted from Gentianaceae, ${ }^{11}$ relatively little is known about the anticancer effects of STM and its underlying molecular mechanisms.

In our previous studies, we discovered that STM suppresses HCC cell proliferation. ${ }^{12}$ We also conducted a microarray analysis to identify the underlying mechanism. ${ }^{12}$ FRAT1 was found to be a significantly downregulated gene in STM-treated HepG2 cells.

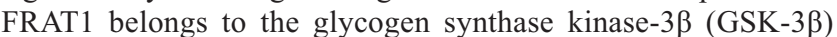
binding protein family and promotes the $\mathrm{Wnt} / \beta$-catenin signaling pathway. However, in the previous report, STM was not found to directly act on the $\mathrm{Wnt} / \beta$-catenin signaling pathway. In the present research, we investigated the anticancer function of STM in two HCC cell lines. We report that STM inhibits proliferation, migration, and invasion of HCC cells by downregulating the FRAT1/Wnt/ $\beta$ catenin signaling axis.

\section{Materials and Methods}

\section{Reagents and cell culture}

We purchased swertiamarin (purity $>95 \%$ ) from Sigma Aldrich (St. Louis, MO, USA). Human hepatoma HepG2 and Huh7 cells were acquired from the Kunming Institute of Zoology. The culture medium was Dulbecco's Modified Eagle's Medium (DMEM) containing 1\% penicillin and $10 \%$ fetal bovine serum (FBS). HCC cells were maintained in a wet incubator in a $5 \% \mathrm{CO}_{2}$ atmosphere at $37^{\circ} \mathrm{C}$. In
Table 1, the antibodies and the dilutions used for Western blot are reported.

\section{Cell viability assay}

The CCK8 (CK04, Dongren Chemical, Japan) assay was used to measure viable cells. 5000 cells were placed in each well of a 96well plate and incubated with DMSO or different concentrations of STM. After $12 \mathrm{~h}$, the CCK 8 mix was added. After a further $4 \mathrm{~h}$, each well absorption values were measured using a microplate reader at a $450 \mathrm{~nm}$ wavelength.

\section{Plate cloning experiment}

A plate cloning experiment was used to detect cell proliferation. A $3 \mathrm{~mL}$ cell suspension (1000 cells) was seeded into 6-well plates. Colony formation occurred within 7 days. After culturing for 7 days, we observed the cells fixed with $4 \%$ paraformaldehyde stained with crystal violet through a microscope.

\section{Nude mouse model}

The animal experiment was approved by the Hospital Ethics Committee of the Second Affiliated Hospital of Kunming Medical University. Four- to eight-week-old male athymic BALB/c nude mice (No. SCXK-2016-0006) were acquired from the Experimental Animal Center of Kunming Medical University and complied with the National Institutes of Health standard. Mice were injected subcutaneously with $1 \times 10^{7} \mathrm{HepG} 2$ cells. Mice in both groups were randomly assigned, the control group $(n=6)$ was injected intratumorally with normal saline, and the treatment group $(n=6)$ received an intratumoral injection of $10 \mu \mathrm{g}$ STM. The living conditions of mice were observed every day, and the length and diameter of tumors in mice were measured with vernier caliper every week. The tumor volume was calculated according to the formula $\mathrm{V}=\mathrm{a} \times \mathrm{b}^{2} / 2$ ( $\mathrm{a}$ was the longest diameter of the tumor and $\mathrm{b}$ was the shortest diameter of the tumor). After 8 weeks of treatment, animals were sacrificed and tumors were excised to measure weight.

\section{Cell proliferation assays by RTCA}

An xCELLigence Real-Time Cell Analyzer (ACEA Biosciences, San Diego, CA, USA) was used for analyzing HepG2 and Huh7 cell proliferation. Each well of an E-plates 16 (ACEA Biosciences) was filled with 3000 cells and monitored for $24 \mathrm{~h}$. When the cells entered the log phase, $10 \mu \mathrm{L}$ of STM was added in two independent experiments and monitored in the RTCA instrument for $72 \mathrm{~h}$.

\section{Cell migration and invasion assay}

In the migration assay, we placed $200 \mu \mathrm{L}$ serum-free cell suspension $\left(1 \times 10^{5}\right.$ cells $)$ in the upper transwell chambers. In the

Table 1. Antibodies used for Western blot.

\begin{tabular}{ll} 
Name & Antibody \\
\hline FRAT1 & Primary antibody \\
$\beta$-catenin & $1: 500$, OM164801, Omnimabs Alhambra, CA USA \\
\hline p- $\beta$-Catenin & ab32572, 1:1000, Abcam, Cambridge, MA, USA \\
GSK-3 $\beta$ & ab11350, 1:500, Abcam, Cambridge, MA, USA \\
\hline CyclinD1 & 12456 S, 1:1000, Cell Signaling Technology, Danvers, MA, USA \\
GAPDH & ab134175, 1:2000, Abcam, Cambridge, MA, USA \\
\hline & ab8245, 1:10,000, Abcam, Cambridge, MA, USA \\
\hline Goat anti-rabbit & Secondary antilbody \\
Goat anti-mouse & ab6721, 1:5000, Abcam, Cambridge, MA, USA \\
\hline
\end{tabular}


invasion assay, we first coated the upper transwell chambers with Matrigel (BD Biosciences, San Jose, CA, USA), then seeded the same numbers of cells. We placed $800 \mu \mathrm{L}$ medium with $20 \%$ FBS in the lower chamber. After culturing for $48 \mathrm{~h}$, we removed the cells on the upper surface. Then we observed the cells fixed with $4 \%$ paraformaldehyde stained with crystal violet through a microscope.

\section{Cell migration and invasion assay by RTCA}

We treated cells with STM for $24 \mathrm{~h}$. Then, the medium was changed to serum-free medium for $6 \mathrm{~h}$, and we prepared a $1 \times 10^{4} / 90$ $\mu \mathrm{L}$ cell suspension. In the migration assay, $165 \mu \mathrm{L}$ of $20 \% \mathrm{FBS}$ medium was used to fill the lower chamber. Then, the two chambers were fastened and $90 \mu \mathrm{L}$ of the serum-free medium was placed into the upper chamber. The CIM-plates 16 (ACEA Biosciences) was placed into the RTCA instrument and left to equilibrate for $1 \mathrm{~h}$. Each well of the CIM-plate 16 was filled with $90 \mu \mathrm{L}$ of cell suspension and monitored in the RTCA instrument for $96 \mathrm{~h}$. In the invasion assay, we first coated the upper transwell chambers with Matrigel, then seeded the same numbers of cells.

\section{Microarray}

After HepG2 cells were treated with DMSO or STM, total RNA from each sample was isolated using Trizol (Invitrogen, Carlsbad, CA, USA) and purified using mirVana miRNA Isolation
Kit (Ambion, Austin, TX, USA) following the manufacturer's instructions. RNA integrity was determined by denatured agarose gel electrophoresis. DNA microarray was performed according to standard protocol. CapitalBio cRNA Amplification and Labeling Kit (CapitalBio, BeiJing, China) was used to produce fluorescent dye labeled cDNA. The labeled cDNAs were then hybridized to Agilent human mRNA Array which was designed with eight identical arrays per slide $\left(8 \times 60 \mathrm{~K}\right.$ format). After hybridization at $42^{\circ} \mathrm{C}$ overnight, the arrays were washed with $2 \mathrm{x}$ SSC and $0.2 \%$ SDS at $42^{\circ} \mathrm{C}$ for $5 \mathrm{~min}$, followed by washing with $0.2 \mathrm{xSC}$ and $0.2 \%$ $\mathrm{SDS}$ at room temperature for $5 \mathrm{~min}$. The array data were analyzed for data summarization, normalization and quality control by using the GeneSpring software V13 (Agilent, Carpinteria, CA, USA). To select the differentially expressed genes, we used threshold values of $\geq 2$ and $\leq-2$-fold change and Benjamini-Hochberg corrected $\mathrm{p}$ value of 0.05 . The data were $\log 2$ transformed and median centered by genes using the Adjust Data function of CLUSTER 3.0 software. Finally, tree visualization was performed using Java Treeview (Stanford University School of Medicine, Stanford, CA, USA)

\section{Lentivirus infection and generation of stable cell lines}

The lentivirus with FRAT1 cDNA (over- FRAT1) was produced by OBIO (Shanghai, China). The cDNA sequences of FRAT1
A

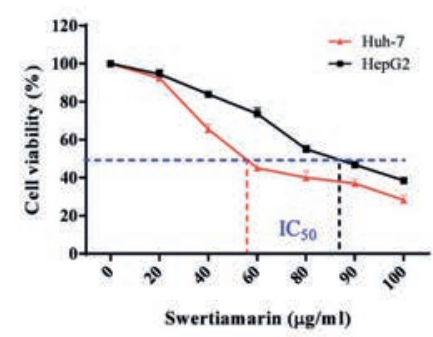

C

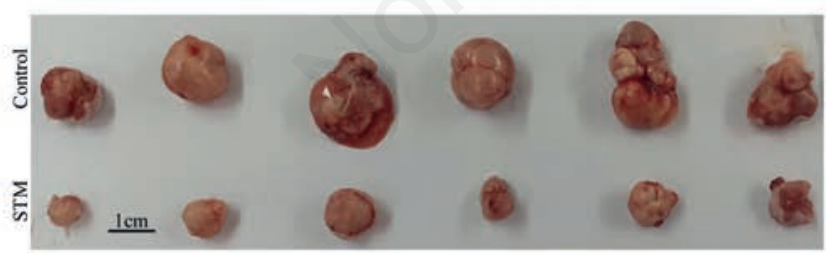

B

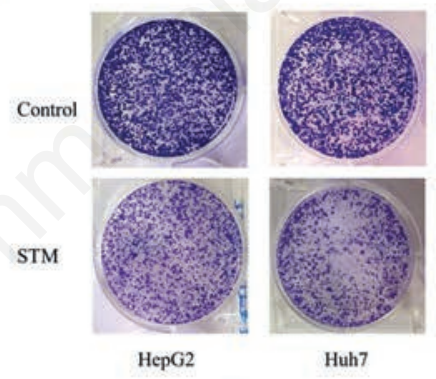

D

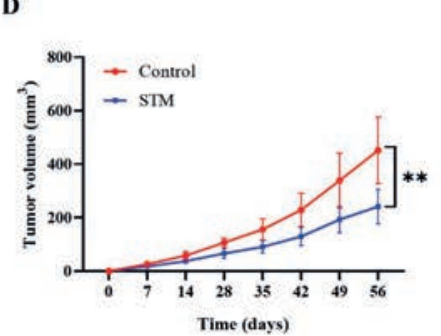

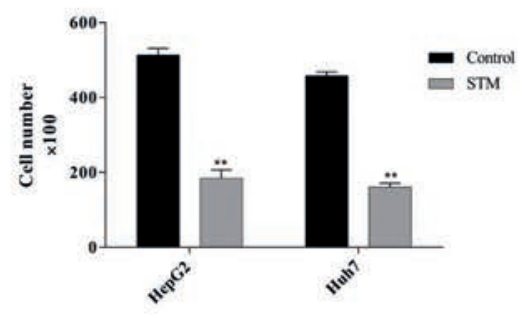

E

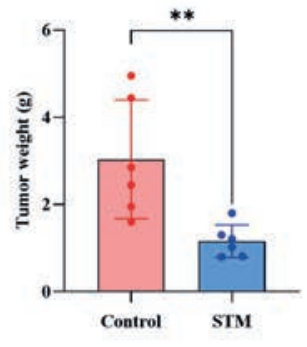

$\mathbf{F}$

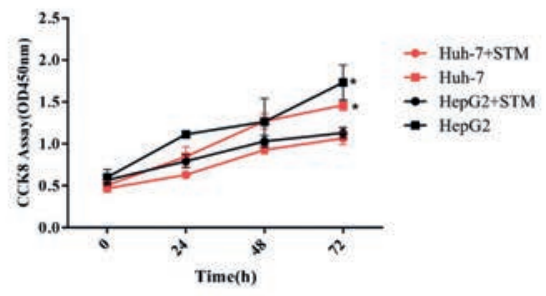

G

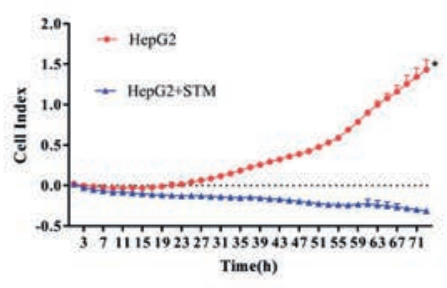

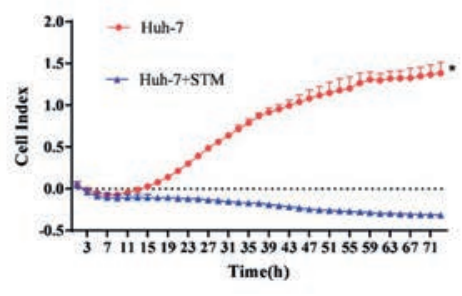

Figure 1. STM suppressed the growth of HCC. A) Cell viability assays in HCC cells exposed to the indicated doses of STM. B) Effect of STM on colony formation in HepG2 and Huh7. C) Tumor tissues of nude mice. D) Tumor volumes of two nude mice group. E) Tumor weights of two nude mice group. F) Cell proliferation determined by CCK8 assay. G) Cell proliferation determined by RTCA analysis. ${ }^{*} \mathrm{p}<0.05,{ }^{* *} \mathrm{p}<0.01$ 
(NCBI, NM_005479.4) were incorporated by OBIO. FRAT1 level was identified by RT-PCR and Western blot.

\section{RNA extraction and qRT-PCR}

The entire RNA of HepG2 and Huh7 cells was separated using TRIZOL reagent (Invitrogen) following the protocol. For the reverse-transcription of RNA, we used the PrimeScript ${ }^{\mathrm{TM}}$ RT reagent Kit (Takara, Dalian, China) with gDNA Eraser by the protocol. Quantitative real-time PCR was executed on a CFX96 Touch $^{\mathrm{TM}}$ Real-Time PCR Detection System (Bio-Rad Laboratories, Hercules, CA, USA) with SYBR Premix Ex Taq (Takara, Dalian, China). We normalized mRNA transcription levels with GAPDH expression. Table 2 displays the sequences of the primers. The mRNA values of FRAT1 and $\beta$-catenin were calculated using the $2^{-\Delta \Delta \mathrm{Ct}}$ method.

\section{Western blot}

We used RIPA lysis buffer to extract the total protein. We supplemented it with protease inhibitor and phosphatase inhibitor and separated it using $10 \%$ sodium lauryl sulfate-polyacrylamide gel electrophoresis. After transfer to a $0.22 \mu \mathrm{m}$ polyvinylidene difluoride (PVDF) membrane, the membrane was incubated with $5 \%$ bovine serum albumin for $1 \mathrm{~h}$ and then with a primary antibody (Table 1 ) at $4{ }^{\circ} \mathrm{C}$ overnight. The membrane was washed for 5 min 3 times in Tristan Buffered saline Tween (TBST), then were cultivated with the secondary antibody (Table 1 ) for $1 \mathrm{~h}$. Protein bands were analyzed using Image-Pro Plus 6.0.

\section{Statistical analysis}

We performed each experiment at least three times. The data of the two samples were compared using the Student's $t$-test. The data of the multiple samples were compared using the one-way analysis of variance. Data were expressed as mean \pm SD. Values of $p<0.05$ were considered statistically significant.

\section{Results}

\section{STM significantly suppresses the growth of HCC}

To test STM's action on HCC cell growth, we exposed HCC cells to STM in different concentrations for 24-120 h. STM

Table 2. Primers used for qRT-PCR.

\begin{tabular}{ll} 
Gene & \multicolumn{1}{c}{ Sequence } \\
FRAT1 & Upper primer: 5 -CAGGCCGACCTTGATGGG-3 \\
& Lower primer: 5 -TGGGGAAGCTTTGCACGTAA-3 \\
p-catenin & Upper primer: 5 -AACTTGCCACACGTGCAATC -3 \\
& Lower primer: 5 -GGTTATGCAAGGTCCCAGC-3 \\
\hline GAPDH & Upper primer: 5 -GACAGTCAGCCGCATCTTCT-3 \\
& Lower primer: 5 -TTAAAAGCAGCCCTGGTGAC-3 \\
\hline
\end{tabular}

B
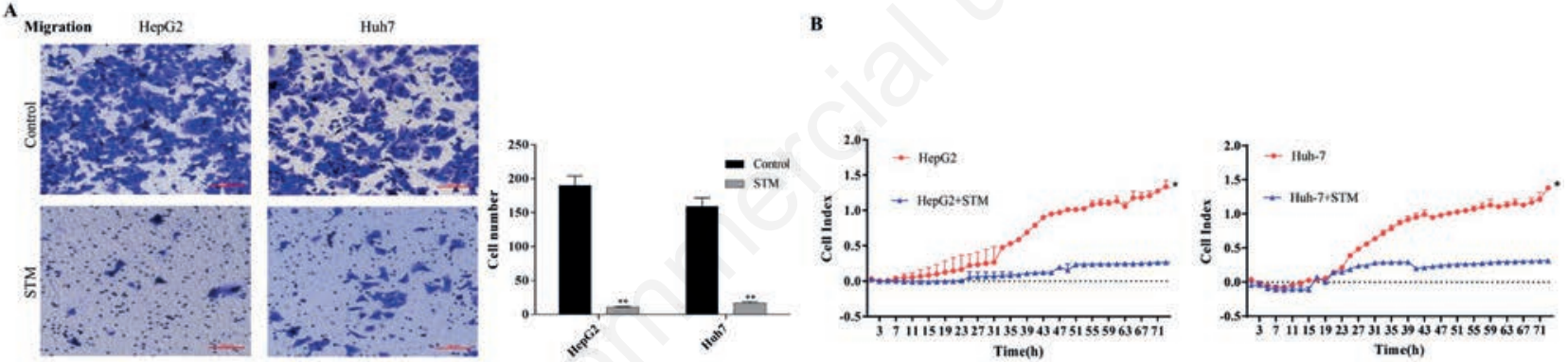

D
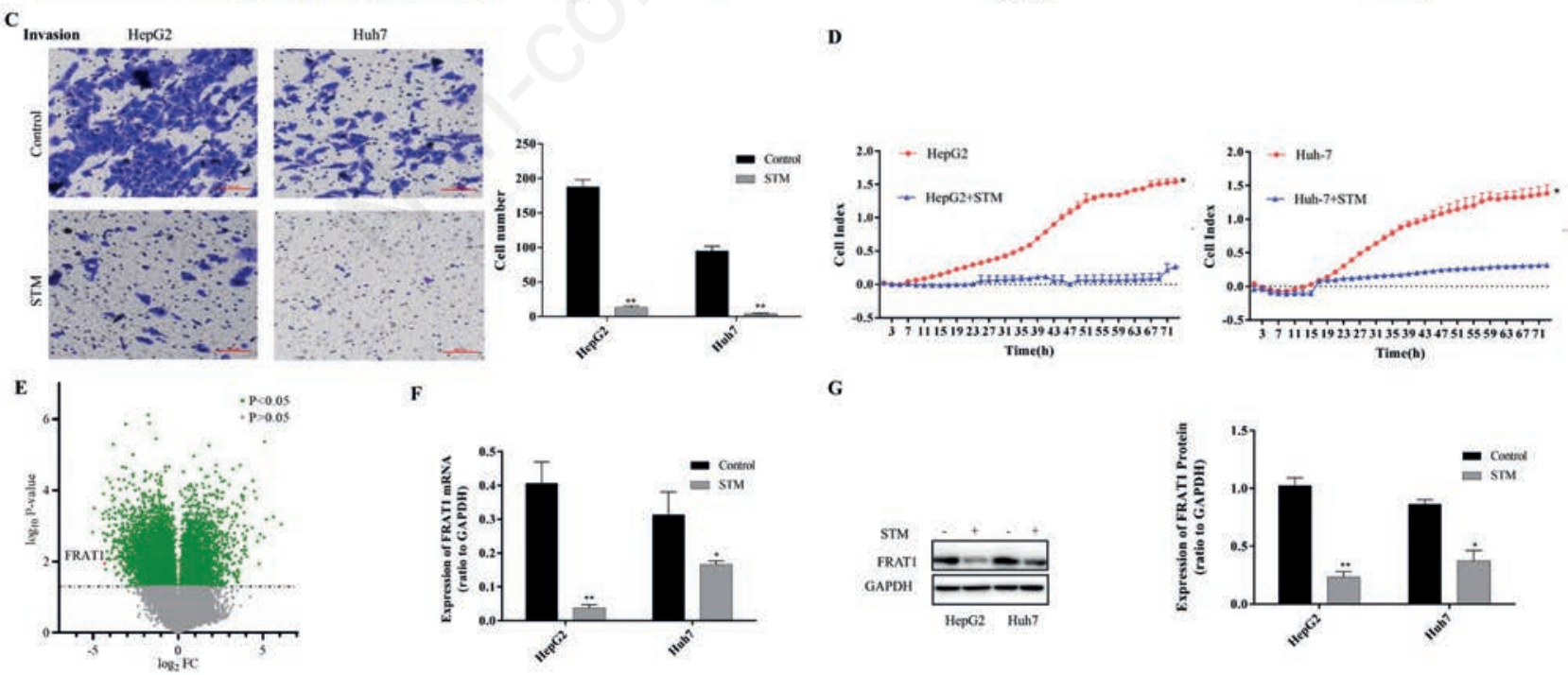

F

G
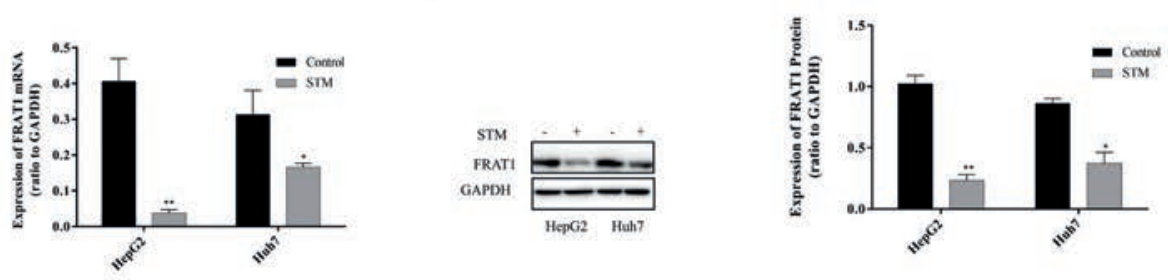

Figure 2. STM decreased HCC cell migration and invasion, and negatively regulates FRAT1. A) Cell migration determined using transwell assays; scale bar: $100 \mu \mathrm{m}$. B) Cell migration determined using RTCA assays. C) Cell invasion determined using transwell assays; scale bar: $100 \mu \mathrm{m}$. D) Cell invasion determined using RTCA assays. E) Differentially expressed genes (DEGs) were assessed after the STM treatment of HepG2 cells; the red dot is the FAT1 gene, which is a significantly DEG; the red dot FRAT1 was p=0.01, $\log 2 F C=$ -4.32. F) FRAT1 mRNA level by qRT-PCR assay in HCC cells treated with STM. G) The protein level of FRAT1 in HCC cells treated with STM determined from Western blot analysis. ${ }^{*} \mathrm{p}<0.05,{ }^{* *} \mathrm{p}<0.01$. 
suppressed HCC cell proliferation in a dose-dependent manner. The $\mathrm{IC}_{50}$ of STM were $87.96 \pm 1.408 \mu \mathrm{g} / \mathrm{mL}$ (HepG2) and $56.49 \pm 0.759$ (Huh7) $\mu \mathrm{g} / \mathrm{mL}$ (Figure 1A). Figure 1B shows that $70 \mu \mathrm{g} / \mathrm{mL}$ (HepG2) and $50 \mu \mathrm{g} / \mathrm{mL}$ (Huh7) STM significantly shortened the number and size of colonies formed by HCC cells after seven days of culture. Figure 1C-E show that STM significantly reduced the volume and weight of the tumor in nude mice.

\section{STM decreases proliferation, migration, and invasion of HCC cells}

Cell proliferation was observed using the CCK8 assay and the RTCA system separately. Cell migration and invasion were detected using transwell assay and the RTCA system. The outcome showed that HCC cell proliferation was significantly reduced by STM $(\mathrm{p}<0.05$, Figures 1 F, G). Figure 2 A-D show that STM significantly reduced HCC cell migration and invasion. These results revealed that STM inhibits HepG2 and Huh7 cell proliferation, migration, and invasion.

\section{STM negatively regulates FRAT1 in HCC cells}

FRAT1 was found to be a significantly downregulated gene in STM-treated HepG2 cells by microarray analysis (Figure 2E). Next, FRAT1 was measured after STM treatment. The result showed that STM downregulated FRAT1 in HepG2 and Huh7 cells (Figure 2 F,G). From this result, we hypothesized that FRAT1 may be involved in STM's suppressive activity on HCC cells.
STM suppresses HCC cell proliferation, migration, and invasion by downregulating FRAT1

To identify the function of STM involving FRAT1 in HCC cells, FRAT1 lentiviral vector (FRAT1-OE) and its corresponding FRAT1 empty lentiviral vector (FRAT1-NC) were transfected into HepG2 and Huh7 cells. Our findings showed that compared with FRAT1NC transfection, transfection with FRAT1-OE promoted cell proliferation, migration, and invasion (Figures $3 \mathrm{~A}-\mathrm{H}$ ). Transfection with FRAT1-OE impaired the antitumor function of STM (Figures 3 A-H). The above results showed that STM suppresses HCC cell proliferation, migration, and invasion through silencing FRAT1.

\section{STM inactivates FRAT1/Wnt/ $\beta$-catenin signaling axis}

Per previous studies, FRAT1 is a positive regulator in the Wnt/ $\beta$-catenin signaling pathway in cancer. To explore STM's function in the FRAT1/Wnt/ $\beta$-catenin signaling axis, we determined the expression of pathway-associated proteins. STM treatment increased the level of $\mathrm{p}-\beta$-catenin and GSK-3 $\beta$ in comparison with the control (Figure 4C), whereas FRAT1, $\beta$-catenin, and Cyclin D1 were all downregulated by STM (Figures 4 A-C). To further confirm these findings, we found that overexpression of transfected FRAT1OE reduced the function of STM in inhibiting the Wnt $\beta$-catenin pathway, which was characterized by a reverse change in the above proteins' expression levels (Figures 4 A-C). In summary, STM inactivates the FRAT1/Wnt/ $\beta$-catenin signaling axis.

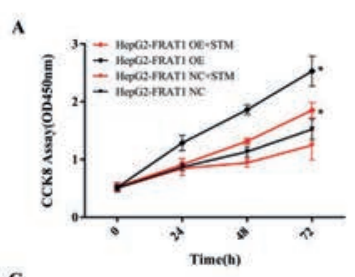

C

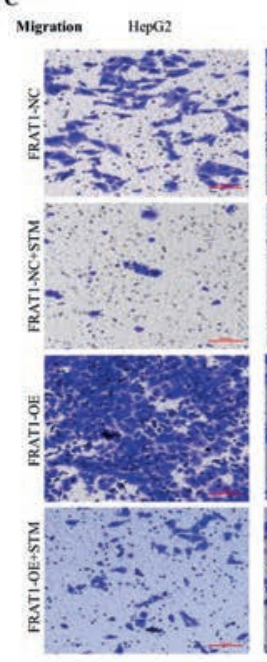

Huh?

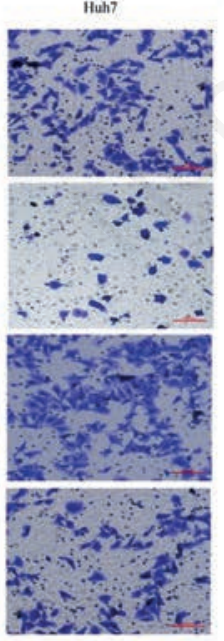

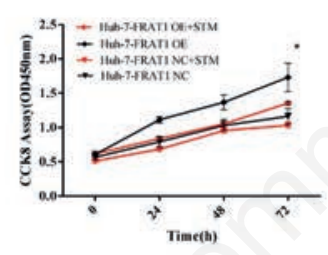

D

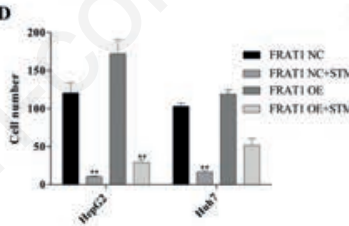

E
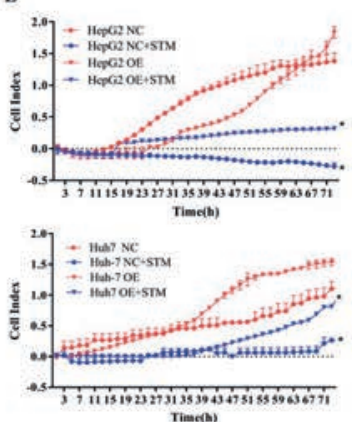

B
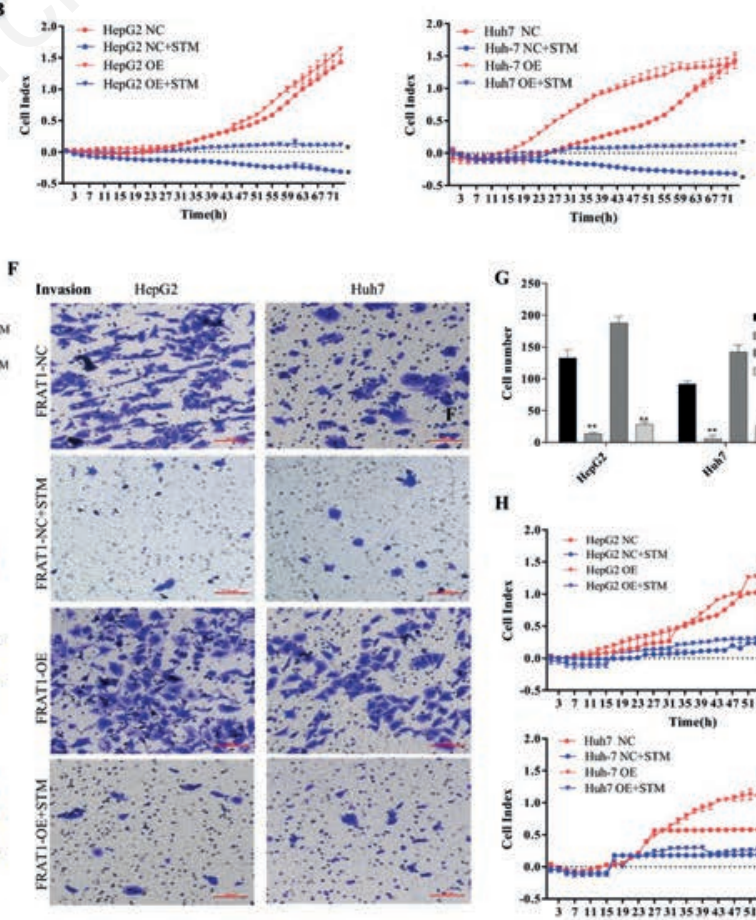

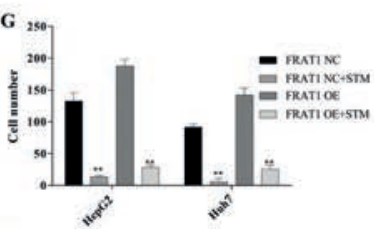

H
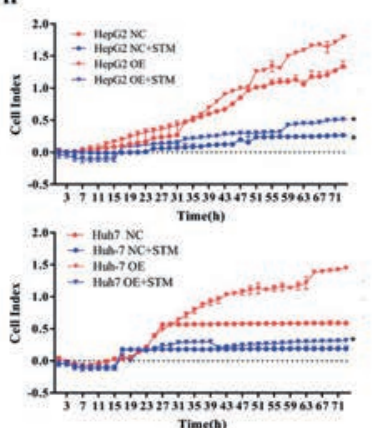

Figure 3. STM suppresses HCC cell proliferation, migration, and invasion by silencing FRAT1. A) Cell proliferation by CCK8 analysis in cells over-expressing FRAT1 treated with STM. B) Cell proliferation per RTCA assay in over-expressing FRAT1 cells treated with STM. C,D) Cell migration per the transwell assays in over-expressing FRAT1 cells treated with STM; scale bar: $100 \mu \mathrm{m}$. E) Cell migration per the RTCA assays in over-expressing FRAT1 cells treated with STM. F,G) Cell invasion by the transwell assays in over-expressing FRAT1 cells exposed to STM; scale bar: $100 \mu \mathrm{m}$. H) Cell invasion by the RTCA assays in over-expressing FRAT1 cells exposed to STM. ${ }^{*} \mathbf{p}<0.05,{ }^{* *} \mathbf{p}<\mathbf{0 . 0 1}$. 


\section{Discussion}

HCC is an important disease with high levels of mortality worldwide, with China generating half of the world's total number of cases. ${ }^{13}$ Less than $30 \%$ of patients can undergo surgery because most HCC cases are in the advanced disease stage and not eligible for surgery. ${ }^{14}$ Food and Drug Administration (FDA) approved sorafenib and regorafenib for HCC therapy are expensive and fail to achieve a satisfactory response rate..$^{15,16}$ In this case, exploring a rational combination of therapies based on the individual characteristics of HCC patients is still necessary, including complementary and alternative medicines. ${ }^{17}$ To date, many researchers have focused on isolating monomers from herbal medicines to find new drugs for HCC treatment. Yang studied the effect of swertiamarin on the proliferation of MGC803 cells in vitro and in vivo. Compared with the control group, swertiamarin at different concentrations could effectively inhibit the proliferation and induce apoptosis of MGC803 cells. ${ }^{18}$ In this study, a new method of HCC treatment was investigated and the mechanism of its anticancer activity was studied. It was found that STM downregulates the FRAT1/Wnt/ $\beta$-catenin signaling axis to inhibit the proliferation, migration, and invasion of HCC cells.

Gentianaceae has 87 genera and about 1600 species. Many Gentianaceae plants are commonly used as hepatic protectors in traditional Chinese herbal medicines, Tibetan medicine, and Indian Ayurvedic medicine. ${ }^{19}$ STM is a monomer isolated from the active ingredients of these plants and has shown many biological activities. In an adjuvant arthritis rat model, swertiamarin inhibited the development of arthritis by inhibiting the release of nuclear factor kappa-B. ${ }^{20}$ In the oleic-acid-induced steatosis HepG2 cell model, swertiamarin attenuated oxidative stress by activating Adenosine 5 '-monophosphate-activated protein kinase. ${ }^{21}$ In a rat model of Freund's complete adjuvant-induced arthritis, swertiamarin significantly attenuated mitogen-activated protein kinase release in a dose-dependent manner. ${ }^{22}$ In a rat model of carbon tetrachlorideinduced liver damage, STM therapy improved hepatocyte apoptosis by attenuating the phosphatidylinositol-3-kinases and proteinserine-threonine kinase. ${ }^{23}$ This research focuses on the anticancer effect of STM on HCC. The data indicate that STM has significant concentration-dependent anticancer activity in HCC cells and inhibits tumor growth in vivo. STM has an effective suppression effect on HCC cells.

The classical Wnt/ $\beta$-catenin signaling pathway is known to be related to tumor progression, including in HCC, breast, ovarian, and colorectal cancers. ${ }^{24}$ The nature of WNT signaling is the inhibition and degradation of $\beta$-catenin phosphorylation. $\beta$-catenin accumulates in the cytoplasm and increases nuclear translocation, thereby inducing the transcription of target genes downstream of Wnt, such as Cyclin D1. ${ }^{24}$ In normal cellular homeostasis, the cytoplasmic GSK3 $\beta$ complex phosphorylates $\beta$-catenin and $\mathrm{p}-\beta$ catenin is rapidly degraded. ${ }^{25}$ FRAT1 can promote the Wnt/ $\beta$-catenin signaling pathway since it competes with Axin 1 to bind the GSK3 $\beta$ complex and then inhibits $\beta$-catenin phosphorylation. ${ }^{26}$ FRAT1 is a recognized oncogene, the overexpression of which is associated with adverse clinical outcomes in several cancers, including HCC, ${ }^{27}$ cartilage tumors, ${ }^{28}$ esophageal squamous cell carcinoma, ${ }^{29}$ gliomas, ${ }^{30}$ astrocytoma, ${ }^{31}$ ovarian cancer, ${ }^{32}$ and non-small cell lung cancer. ${ }^{33}$ Our findings revealed that STM reduces FRAT1, $\beta$-catenin, and Cyclin-D1 levels and increases GSK-3 $\beta$ and p- $\beta$-catenin levels in HCC cells, which means that STW regulates the Wnt/ $\beta$-catenin signaling pathway. In STM-treated HCC cells, FRAT1 overexpression led to $\beta$-catenin activation and cyclin D1 expression and attenuated STM inhibition of HCC cell growth. This suggests that STM's inhibition of HCC cells may occur by suppressing the FRAT1/Wnt/ $\beta$-catenin signaling axis, at least in part.
A
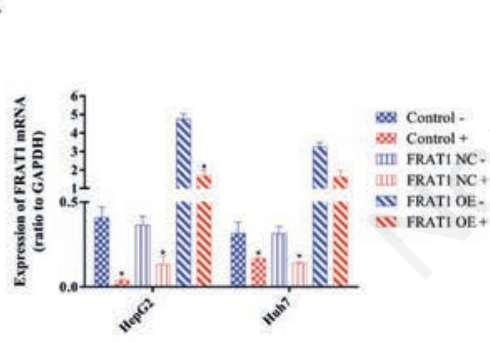

B

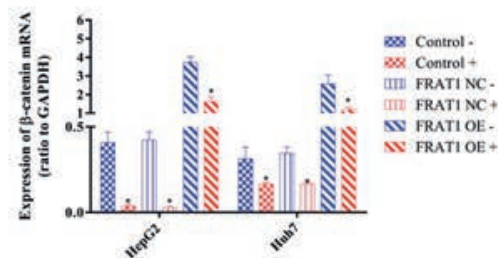

C

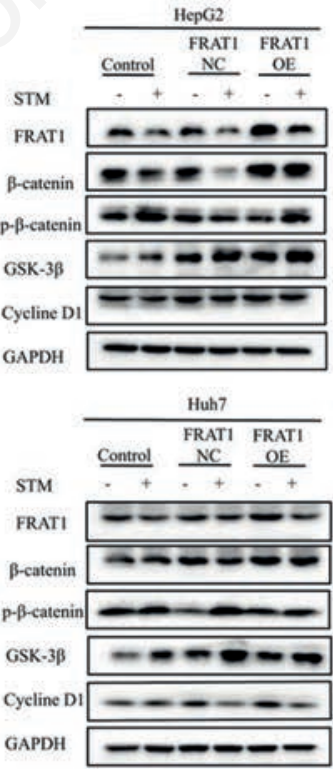

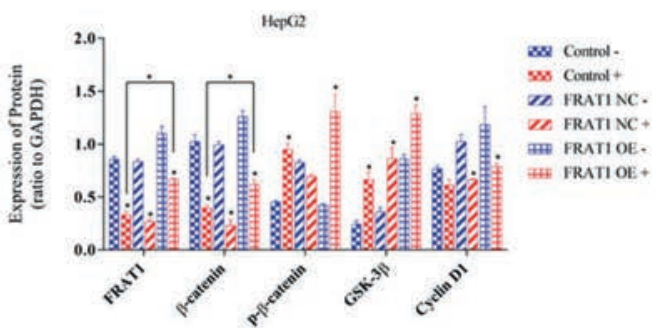

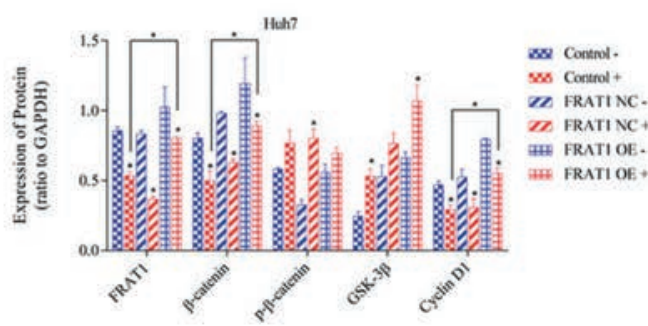

Figure 4. STM inactivates the FRAT1/Wnt/ $\beta$-catenin signaling axis. A) FRAT1 mRNA level per real-time PCR analysis in over-expressing FRAT1 cells treated with STM. B) $\beta$-catenin mRNA level by real-time PCR analysis in over-expressing FRAT1 cells treated with STM. C) Wnt/ $\beta$-catenin signaling pathway marker protein levels determined using Western blot assay in over-expressing FRAT1 cells treated with STM. ${ }^{*} \mathrm{p}<0.05,{ }^{* *} \mathrm{p}<0.01$. 
In brief, our discoveries indicate that STM suppresses Wnt/ $\beta$ catenin signaling pathways in HCC cells at least in part by downregulating FRAT1, leading to inhibition of proliferation, migration, and invasion. These data indicate that STW has a therapeutic potential for $\mathrm{HCC}$.

\section{Acknowledgements}

The authors acknowledge Chen Jijun and Geng Changan of the State Key Laboratory of Phytochemistry and Plant Resources in Western China at the Kunming Institute of Botany, Chinese Academy of Sciences. They participated in our previous research and provided comments on our manuscript.

\section{References}

1. Bray FI, Ferlay J, Soerjomataram I, Siegel RL, Torre LA, Jemal A. Global cancer statistics 2018: GLOBOCAN estimates of incidence and mortality worldwide for 36 cancers in 185 countries. CA-Cancer J Clin 2018;68:394-424.

2. Shen Q, Eun JW, Lee KB, Kim HS, Yang HD, Kim SY, et al. Barrier to autointegration factor 1, procollagen-lysine, 2-oxoglutarate 5-dioxygenase 3 , and splicing factor $3 \mathrm{~b}$ subunit 4 as early-stage cancer decision markers and drivers of HCC. Hepatology 2018;67:1360-77.

3. Villanueva A, Hoshida Y, Battiston C, Tovar V, Sia D, Alsinet $\mathrm{C}$, et al. Combining Clinical, pathology, and gene expression data to predict recurrence of HCC. Gastroenterology 2011;140:1501-12.

4. Ma Y, Ke Y, Zi X, Zhao W, Shi X, Liu H. Jaridonin, a novel ent-kaurene diterpenoid from isodon rubescens, inducing apoptosis via production of reactive oxygen species in esophageal cancer cells. Curr Cancer Drug Targets 2013;13:611-24.

5. Der Nat JMV, Der Sluis WGV, Labadie RP. Gentiogenal, a New antimicrobial iridoid derived from gentiopicrin (Gentiopicroside). Planta Med 1982;45:161-2.

6. Der Sluis WGV, Labadie RP. Fungitoxic activity of the secoiridoid glucoside gentiopicrin (Gentiopicroside). Planta Med 1981;42:139-40.

7. $\mathrm{Wu} \mathrm{X}, \mathrm{Gu}$ Y, Li L. The anti-hyperplasia, anti-oxidative and anti-inflammatory properties of Qing Ye Dan and swertiamarin in testosterone-induced benign prostatic hyperplasia in rats. Toxicol Lett 2017;265:9-16.

8. Sonawane RD, Vishwakarma SL, Lakshmi S, Rajani M, Padh H, Goyal RK. Amelioration of STZ-induced type 1 diabetic nephropathy by aqueous extract of Enicostemma littorale Blume and swertiamarin in rats. Mol Cell Biochem 2010;340:1-6.

9. Jaishree V, Badami S. Antioxidant and hepatoprotective effect of swertiamarin from Enicostemma axillare against D-galactosamine induced acute liver damage in rats. J Ethnopharmacol 2010;130:103-6

10. Jie X, Geng C, Huang X, Ma Y, Zhang X, Zhang R, et al. Five new secoiridoid glycosides and one unusual lactonic enol ketone with anti-HBV activity from Swertia cincta. Fitoterapia 2015;102 96-101.

11. Wani BA, Ramamoorthy D, Rather MA, Arumugam N, Qazi AK, Majeed R, et al. Induction of apoptosis in human pancreatic MiaPaCa-2 cells through the loss of mitochondrial membrane potential $(\Delta \Psi \mathrm{m})$ by Gentiana kurroo root extract and LC-ESI-MS analysis of its principal constituents.
Phytomedicine 2013;20:723-33.

12. Tang H, Ke Y, Ren Z, Lei X, Xiao S, Bao T, et al. Bioinformatics analysis of differentially expressed genes in HCC cells exposed to Swertiamarin. J Cancer 2019;10:652634.

13. Liu X, Li M, Wang X, Dang Z, Jiang Y, Wang X, et al. PD-1 + TIGIT $+\mathrm{CD} 8+\mathrm{T}$ cells are associated with pathogenesis and progression of patients with hepatitis B virus-related HCC. Cancer Immunol Immunother 2019;68:2041-54.

14. Ye SL, Takayama T, Geschwind JH, Marrero JA, Bronowicki JP. Current approaches to the treatment of early HCC. Oncologist 2010;15:34-41.

15. Bruix J, Qin S, Merle P, Granito A, Huang Y, Bodoky G, et al. Regorafenib for patients with HCC who progressed on sorafenib treatment (RESORCE): a randomised, double-blind, placebo-controlled, phase 3 trial. Lancet 2017;389:56-66.

16. Galle PR. Sorafenib in advanced HCC - We have won a battle not the war. J Hepatol 2008;49:871-3.

17. Hong M, Wang N, Tan HY, Tsao SW, Feng Y. MicroRNAs and Chinese medicinal herbs: New possibilities in cancer therapy. Cancers 2015;7:1643-57.

18. Weiqiang Y, Boshun W, Qing C. Inhibitory effect of swertiamarin on MGC803 Cells in vitro and in vivo. Chin J Gastro 2019;2:86-9.

19. Suryawanshi S, Asthana RK, Gupta RC. Assessment of systemic interaction between Swertia chirata extract and its bioactive constituents in rabbits. Phytother Res 2009;23:1036-8.

20. Saravanan S, Islam VIH, Babu NP, Pandikumar P, Thirugnanasambantham $\mathrm{K}$, Chellappandian $\mathrm{M}$, et al. Swertiamarin attenuates inflammation mediators via modulating NF- $\kappa \mathrm{B} / \mathrm{I} \kappa \mathrm{B}$ and JAK2/STAT3 transcription factors in adjuvant induced arthritis. Eur J Pharm Sci 2014;56:70-86.

21. Patel TP, Rawal K, Soni S, Gupta S. Swertiamarin ameliorates oleic acid induced lipid accumulation and oxidative stress by attenuating gluconeogenesis and lipogenesis in hepatic steatosis. Biomed Pharmacother 2016;83:785-91.

22. Saravanan S, Islam VIH, Thirugnanasambantham K, Pazhanivel N, Raghuraman N, Paulraj MG, et al. Swertiamarin ameliorates inflammation and osteoclastogenesis intermediates in IL-1 $\beta$ induced rat fibroblast-like synoviocytes. Inflamm Res 2014;63:451-62.

23. Zhang Q, Chen K, Wu T, Song H. Swertiamarin ameliorates carbon tetrachloride-induced hepatic apoptosis via blocking the PI3K/Akt pathway in rats. Korean J Physiol PHA 2019;23:21-8.

24. Pendás-Franco N, Aguilera O, Pereira F, González-Sancho JM, Muñoz A. Vitamin D and Wnt/B-catenin pathway in colon cancer: Role and regulation of DICKKOPF genes. Anticancer Res 2008;28:2613-23.

25. Jamieson C, Sharma M, Henderson BR. Wnt signaling from membrane to nucleus: $\beta$-catenin caught in a loop. Int $\mathrm{J}$ Biochem Cell B 2012;44:847-50.

26. Fraser E, Young N, Dajani R, Francakoh J, Ryves J, Williams RSB, et al. Identification of the Axin and Frat binding region of glycogen synthase kinase-3. J Biol Chem 2002;277:217685.

27. Fan W, Du F, Liu X, Chen N. Knockdown of FRAT1 inhibits hypoxia-induced epithelial-to-mesenchymal transition via suppression of the Wnt/ $\beta$-catenin pathway in HCC cells. Oncol Rep 2016;36:2999-3004.

28. He L, Yang Z, Zhou J, Wang W. The clinical pathological significance of FRAT1 and ROR2 expression in cartilage tumors. Clin Transl Oncol 2015; 17:438-45.

29. Wang Y, Liu S, Zhu H, Zhang W, Zhang G, Zhou X, et al. FRAT1 overexpression leads to aberrant activation of $\beta$ - 
catenin/TCF pathway in esophageal squamous cell carcinoma. Int J Cancer 2008;123:561-8.

30. Guo G, Zhong C, Liu Y, Mao X, Zhang Z, Jin J, et al. Overexpression of FRAT1 is associated with malignant phenotype and poor prognosis in human gliomas. Dis Markers 2015;2015:289750.

31. Guo G, Liu B, Zhong C, Zhang X, Mao X, Wang P, et al. FRAT1 expression and its correlation with pathologic grade, proliferation, and apoptosis in human astrocytomas. Med
Oncol 2011;28:1-6.

32. Wang Y, Hewitt SM, Liu SS, Zhou XX, Zhu H, Zhou C, et al. Tissue microarray analysis of human FRAT1 expression and its correlation with the subcellular localisation of $\beta$-catenin in ovarian tumours. Br J Cancer 2006;94:686-91.

33. Zhang $Y$, Yu J, Lin X, Miao Y, Han Y, Fan C, et al. Overexpression of Frat1 correlates with malignant phenotype and advanced stage in human non-small cell lung cancer. Virchows Arch 2011;459:255-63.

Received for publication: 4 August 2020. Accepted for publication: 30 September 2020

This work is licensed under a Creative Commons Attribution-NonCommercial 4.0 International License (CC BY-NC 4.0).

(C) Copyright: the Author(s), 2020

Licensee PAGEPress, Italy

European Journal of Histochemistry 2020; 64:3169

doi:10.4081/ejh.2020.3169 